\title{
LOW LEVEL LASER THERAPY (LLLT) - AN INTRODUCTION TO COLD LASER
}

Samith Ahmed ${ }^{1}$

\section{HOW TO CITE THIS ARTICLE:}

Samith Ahmed. "Low level laser therapy (LLLT) - an introduction to cold laser". Journal of Evolution of Medical and Dental Sciences 2013; Vol2, Issue 39, September 30; Page: 7414-7417.

KEYWORDS: Laser, ATP, Mitochondria, Wound.

\section{WHAT IS LASER}

The word "laser" is an acronym for Light Amplification by the Stimulated Emission of Radiation. The theory was first described in 1916 by Albert Einstein who paved the way for the development of the therapeutic laser that we use today for pain.

HISTORY: In 1967 a Hungarian physicist named Dr. Endre Mester accidentally discovered that a monochromatic laser could help speed up the healing of soft tissue injuries. He took some mice, shaved the hair off their backs, divided them into two groups and gave a laser treatment with a low powered ruby laser to one group. They did not get cancer and to his surprise the hair on the treated group grew back more quickly than the untreated group. That was how "laser bio stimulation" was discovered.

CELLULAR PATHOLOGY: Every eukaryotic cell in an animal's body has one or many thousand cell component called the mitochondrion. These mitochondria are responsible for providing most of the required ATP for cells. ATP is the chemical responsible for energy release within cells that drives a multitude of cellular and physiological functions including those directly related to injury repair and pain relief. When a cell is damaged through injury or trauma, the mitochondrion, figuratively speaking, curls up like a hedgehog. Once this happens, the production of ATP is drastically reduced, or even ceased. As a result, the rate of healing slows dramatically. Cells exposed to infra-red light (Low Level Laser Therapy) at the right frequency, causes the mitochondrion to spring into action almost immediately producing increased amounts of ADP. The ADP then links with free oxygen singlet to produce ATP. Again, Cold laser increases the production of ATP in damaged or resting mitochondria.

Depletion of ATP: The major causes of ATP depletion are reduced supply of oxygen and nutrients, mitochondrial damage, and the actions of some toxins (e.g., cyanide).

Depletion of ATP to $5 \%$ to $10 \%$ of normal levels has widespread effects on many critical cellular systems.

Mitochondrial Damage: Mitochondria are the cell's suppliers of life-sustaining energy in the form of ATP, but they are also critical players in cell injury and death.

Mitochondria can be damaged by increased levels of cytosolic Ca2+

Factors Affecting Wound Healing: Nutrition has profound effects on wound healing. Protein deficiency, for example, and particularly vitamin $\mathrm{C}$ deficiency, inhibit collagen synthesis and retard healing. 
Metabolic status can change wound healing. Diabetes mellitus, for example, is associated with delayed healing, as a consequence of the microangiopathy that is a frequent feature of this disease.

Circulatory status can modulate wound healing. Inadequate blood supply, usually caused by arteriosclerosis or venous abnormalities (e.g., varicose veins) that retard venous drainage, also impairs healing.

Hormones such as glucocorticoids have well-documented anti-inflammatory effects that influence various components of inflammation. These agents also inhibit collagen synthesis.

Infection is the single most important cause of delay in healing, because it results in persistent tissue injury and inflammation.

Mechanical factors, such as early motion of wounds, can delay healing, by compressing blood vessels and separating the edges of the wound.

Low Level Laser Properties: Laser light has unique physical properties, which no ordinary light has. Low Level Laser Therapy increases the speed, quality and strength of tissue repair, resolves inflammation and provides pain relief. Laser therapy has been found to offer superior healing and pain relieving effects compared to other electrotherapeutic modalities such as ultrasound, especially in chronic problems and in the early stages of acute injuries. LLLT is a non-invasive light source treatment that generates a single wavelength of light. It emits no heat, sound, or vibration. It is also called photobiology or biostimulation. LLLT is believed to affect the function of connective tissue cells (fibroblasts), accelerate connective tissue repair and act as an anti-inflammatory agent. Lasers with different wavelengths, varying from 632 to $904 \mathrm{~nm}$, are used in the treatment of musculoskeletal disorders. The Cold laser is a revolutionary medical therapy laser utilizing dual gallium aluminum arsenide lasers specifically engineered for the highly therapeutic near-infrared wavelengths. These wavelengths have significant unique properties for cell stimulation and pain suppression. The K-laser is a medical breakthrough therapy device with unparalleled applications and treatment outcomes.

\section{What LLLT Does?}

LLLT promotes healing in many conditions because it penetrates the skin, increases the ATP and activates enzymes in the targeted cells. Promotes Growth factor response within the cells and tissue as a result of increased ATP and protein synthesis

Improves cell proliferation.

Provides pain relief due to increased endorphin release.

Detoxifies the body to quit addiction.

Strengthens the immune system response via increasing levels of lymphocyte activity.

Current Uses: LLLT is being widely used in biomedicine for the treatment of a variety of skin injuries such as burns, surgical wounds, diabetic wounds and ulceration. Increased ATP production, increased mitochondrial membrane potential and its activity ,transformation of fibroblasts into myofibroblasts increased cell proliferation cell differentiation and collagen production are the key biological effects witnessed following LLLT. Histological inspections of the wound tissue biopsy indicated the formation of new collagen after the laser treatments. 


\section{REVIEW ARTICLE}

Electron microscopy, X-ray diffraction biochemical assay, High Performance Liquid Chromatography (HPLC) and histological analysis are the presently available methods to study collagen deposition. Histopathological examination of the tissue often considered as "gold standard" for the identification of the various pathological stages during the disease progression.

Low-level laser therapy (LLLT) has a wavelength dependent capability to alter cellular behavior in the absence of significant thermal effects. To date, LLLT has been used for therapeutic purposes in treating wound healing, musculoskeletal pain and dental diseases.
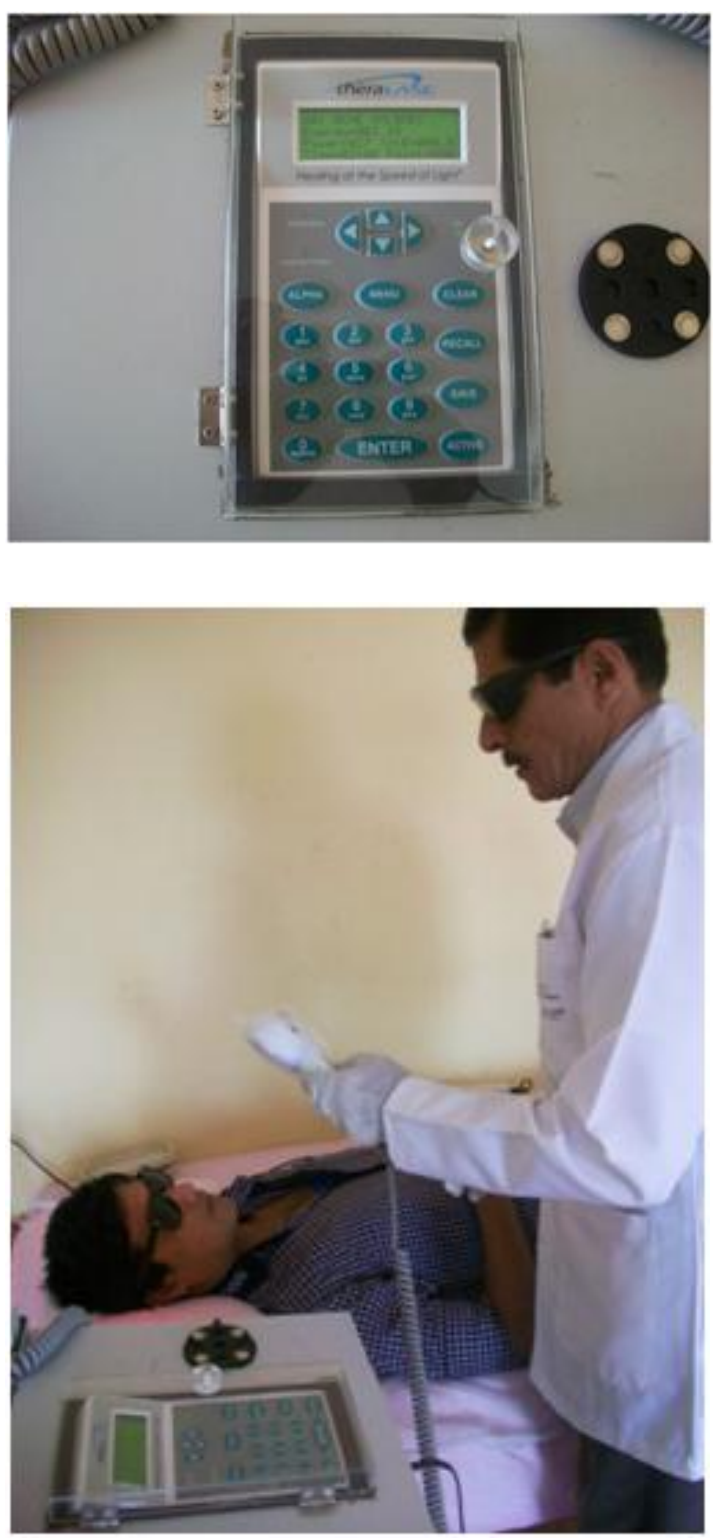

Picture 1: Theralase Cold Laser Machine with 180 inbuilt Protocols
Picture. 2: Safety precautions and protective wear with Multi-LED probe. 


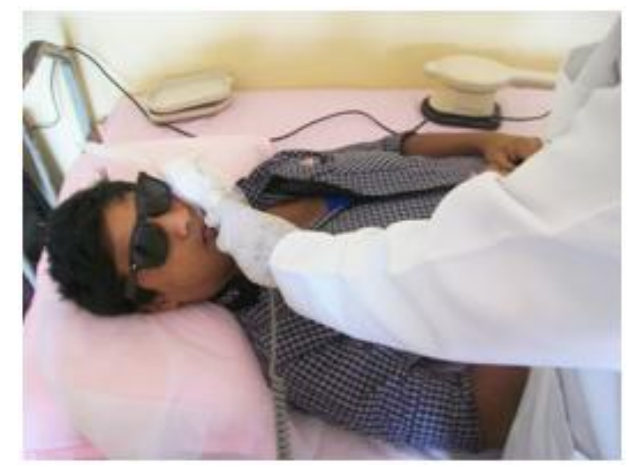

\section{BIBLIOGRAPHY:}

1. Comparison of four non-invasive rewarming methods for mild hypothermia. Daanen, H. A. and Van de Linde, FJ. 12, s. l. Aviat Space Environ Med, 1992, Vol. 63, pp. 1070-1076.

2. Transcutaneous electrostimulation for osteoarthritis of the knee. Rutjes, AW, et al. 4, Oct 2009, Cochrane Database Syst Rev, Vol. 7.

3. Tennis elbow. Clinical Evidence. Bisset, L, Coombes, B and Vicenzino, B. [Online] june 27, 2011. [Cited: June 27, 2009.] http://clinicalevidence. bmj. com/ceweb/index. jsp.

4. Safety of Lase Products user's guide. s. l. Commission, International Electro technical. IEC International Electro technical Commission, 2004. TR 60825-14.

5. Low power laser treatment in patients with knee osteoarthritis. Tascioglu, F, et al. 17-18, May 1, 2004, Swiss Med Wkly, Vol. 134, pp. 254-258.

6. In vivo effects of low level laser therapy on inducible nitric oxide synthase. Moriyama $\mathrm{Y}$, Nguyen J, Akens M, Moriyama EH, Lilge L. 3, March 2009, Lasers Surg Med, Vol. 41, pp. 227231.

7. Photo dissociation of cytochrome c oxidase-nitric oxide complexes. Gorren AC, Van Gelder BF, Wever R. 1988, Ann N Y Acad Sci, Vol. 550, pp. 139-149.

8. Mitochondrial nitric oxide synthase: current concepts and controversies. Lacza Z, Pankotai E, Busija DW. 14, Jan 2009, Front Biosci, Vol. 1, pp. 4436-4443.

9. Nitric oxide and mitochondria. GC. Brown. 12, Jan 2007, Front Biosci, Vol. 1, pp. 1024-1033.

10. Redox signaling (cross-talk) from and to mitochondria involves mitochondrial pores and reactive oxygen species. A, Daiber. 6-7, Jul 2010, Biochim Biophys Acta, Vol. 1797, pp. 897906.

\section{AUTHORS:}

1. Samith Ahmed

\section{PARTICULARS OF CONTRIBUTORS:}

1. Consultant Physician, Department of Alternative Medicine, Sree Sanjeevini Solutions.

\section{NAME ADDRESS EMAIL ID OF THE CORRESPONDING AUTHOR: \\ Dr. Samith Ahmed, Gulistan, K.E. Compound, Mulihitlu Road, Bolar, Mangalore - 575001. \\ Email-samith_17@yahoo.com}

Date of Submission: 03/09/2013.

Date of Peer Review: 07/09/2013.

Date of Acceptance: 20/09/2013.

Date of Publishing: 24/09/2013 